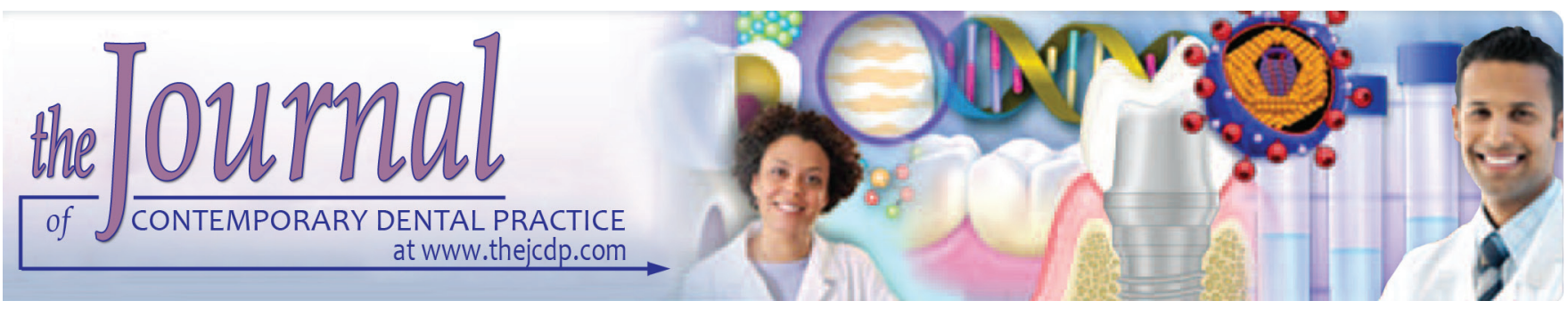

\title{
Botulinum Toxin for the Treatment of Gummy Smile
}

\author{
${ }^{1}$ Afnan F Al-Fouzan, ${ }^{2}$ Lamia S Mokeem, ${ }^{3}$ Reem T Al-Saqat, ${ }^{4}$ Maisa A Alfalah, ${ }^{5}$ Mana A Alharbi, ${ }^{6}$ Abdullah E Al-Samary
}

\section{ABSTRACT}

Aim: The aim was to evaluate the effect of botulinum toxin (Botox) injections as a conservative treatment for gummy smile.

Materials and methods: An experimental in vivo study was conducted at a dermatology clinic in Riyadh in January 2016. The study included 23 female patients who ranged from 20 to 50 years and were treated with Botox injections due to excessive maxillary gingival display. The patients with short clinical crowns or long maxilla, those who were pregnant or breastfeeding, and patients with neuromuscular disorders were excluded. Patients received Botox type I, injected $3 \mathrm{~mm}$ lateral to the alar-fascial groove at the level of the nostril opening at the insertion of the levator labii superioris alaeque nasi muscle. Photos were taken of the patient's smile before and after the treatment and were then uploaded to the SketchUp program to calculate improvements in gingival display. The distance from the lower margin of the upper lip to the gingival margin was calculated pre- and posttreatment. The amount of improvement was calculated as (pre-Botox treatment - post-Botox treatment/pre-Botox treatment $\times 100$ ). The mean percentage of the total improvement was analyzed.

Results: A total of 23 female patients received treatment to improve their gummy smile. Improvement was clear 2 weeks after Botox injection. The mean percentage of improvement in the gingival display was $99.6 \%$.

Conclusion: Botox type I is an effective conservative technique to improve gummy smile caused by muscular hyperfunction.

\footnotetext{
${ }^{1}$ Department of Prosthetic Dental Science, College of Dentistry King Saud University, Riyadh, Kingdom of Saudi Arabia

${ }^{2,3}$ College of Dentistry, King Saud University, Riyadh, Kingdom of Saudi Arabia

${ }^{4}$ Department of Dermatology, King Abdulaziz Medical City Riyadh, Kingdom of Saudi Arabia

${ }^{5}$ Department of Dermatology, College of Medicine, AL-Imam Mohammad Ibn Saud Islamic University, Riyadh, Kingdom of Saudi Arabia

${ }^{6}$ Department of Dermatology, Private Dental Clinic, Riyadh Kingdom of Saudi Arabia

Corresponding Author: Afnan F Al-Fouzan, Department of Prosthodontics, College of Dentistry, King Saud University Riyadh, Kingdom of Saudi Arabia, e-mail: afnan477@hotmail. com; aalfouzan@ksu.edu.sa
}

Clinical significance: Patients' retention highly indicated that they were satisfied with the provided treatment by Botox injections. Improving the quality of life with least painful experience and immediate results was the major advantage for Botox type I.

Keywords: Botulinum toxin, Botulinum toxin type I, Experimental in vivo study, Gummy smile.

How to cite this article: Al-Fouzan AF, Mokeem LS, Al-Saqat RT, Alfalah MA, Alharbi MA, Al-Samary AE. Botulinum Toxin for the Treatment of Gummy Smile. J Contemp Dent Pract 2017;18(6):474-478.

Source of support: This research project was supported by a grant from the "Research Center of the Center for Female Scientific and Medical Colleges," Deanship of Scientific Research, King Saud University.

\section{Conflict of interest: None}

\section{INTRODUCTION}

The 21st century ushered dramatic improvements in quality of life. One of the greatest improvements in the medical field was the emergence of cosmetic services across many countries. This field expanded rapidly as it strongly affects psychological status, and many people believe that cosmetic procedures increased their self-esteem. ${ }^{1}$ Recently, these cosmetic procedures have been introduced to dentistry, with many patients seeking minimally invasive cosmetic procedures. ${ }^{2}$ The smile is considered the most pleasant and complex of all the facial expressions. ${ }^{3}$ It has been said that "a smile is one of the most effective means by which people convey their emotions". ${ }^{4}$ A person's smile depends on three main components: teeth, gums, and lips. Many have studied the components of an attractive smile; most studies concluded that the perfect amount of maxillary gingival tissue shown during smiling is $3 \mathrm{~mm} .{ }^{4}$ Gummy smile, or excessive gingival display in the maxillary arch on smiling, is known as the difference between the lower margin of the upper lip and the superior margin of the incisors ${ }^{1,4,5}$ and is considered one of the 
most embarrassing and self-conscious cosmetic aspects in humans. ${ }^{5}$

Over time, different solutions were proposed to treat this disorder that ranged from complicated to simple. Orthognathic surgeries, such as LeFort I osteotomy and bone resection, orthodontic treatment by incisor intrusion, crown lengthening, gingivoplasty, and botulinum toxin (Botox) injections are all different methods of treating a gummy smile depending on the patient's situation. ${ }^{3,5}$

Botox or Botulinum toxin type I is used as a temporary treatment for gummy smile when it is caused by muscular hyperfunction. ${ }^{1}$ Botulinum is a Gram-positive bacterium that was isolated from Bacillus botulinum by a microbiologist in 1897. In 1990, a dermatologist and ophthalmologist reported its cosmetic effect, which they noticed while treating patients for blepharospasm. ${ }^{2}$ Its first esthetic usage was for the treatment of glabellar frown lines. ${ }^{6}$ Botox type I inhibits acetylcholine release into the synapse by bonding to the nerve at the neuromuscular ending, causing the acetylcholine protein located on the cell membrane to be inhibited. In turn, muscle contraction is inhibited, leading to reversible muscle atrophy. In 1989, the Food and Drug Administration (FDA) approved the use of Botox as a safe and effective therapy for different diseases. In 1990, its use was approved by the National Institutes of Health. ${ }^{4}$

Gummy smile should be first classified either according to the area of the maxilla shown, i.e., anterior, posterior, mixed, or asymmetric, ${ }^{3}$ or via the Goldstein classification as low, medium, or high based on the amount of exposure. ${ }^{4}$ This classification is important to identify the involved muscle and to modify the injection technique of Botox type I. ${ }^{3}$ The effect of Botox will appear after 2 weeks and persists between 4 and 6 months depending on the muscle thickness and anatomy. ${ }^{2}$ The aim of this study was to demonstrate the success of Botox injections as a conservative treatment for gummy smile due to a hyperfunctional muscle in the Saudi community. The null hypothesis was that Botox type I is not effective for the treatment of gummy smile.

\section{MATERIALS AND METHODS}

This study examined 23 patients who met the following inclusion criteria: excessive maxillary gingival display due to muscle hyperfunction. We excluded patients with gummy smile due to short clinical crowns or long maxilla, pregnant patients, or patients who were breastfeeding, as well as patients with neuromuscular disorders. The study was conducted at a private dermatology clinic in Riyadh in January 2016; the included patients received a Botox injection to improve their smile by a consulting dermatologist.

\section{Preinjection Preparation}

Patients signed an informed consent form that was approved by the Ethical Committee of the College of Dentistry Research Center at King Saud University, and the study was conducted in accordance with the principles of the Helsinki Declaration.

Preinjection photos were taken with a Canon EOS 70D (Digital AF / AE single-lens reflex camera with builtin flash, Canon, USA). The patient smiled and sat in a standardized position. The natural head position was a standardized and reproducible position of the head in an upright posture. The eyes were focused on a point in the distance at eye level, which implies that the visual axis is horizontal. ${ }^{7,8}$ Topical anesthesia gel (lidocaine $4 \%$, L.M.X $4^{\circledR}$, MI, USA) was used at the site of injection for 15 to 20 minutes and covered by plastic wrap. An alcohol swab (saturated with $70 \%$ isopropyl alcohol, Freshmed ${ }^{\circledR}$, Jeddah, Kingdom of Saudi Arabia) was used to sterilize the area of injection.

\section{Botox Injection}

A 30-gauge needle (BD Microlance $3^{\circledR}$, Medisave, UK) with a tip (BD $1 \mathrm{~mL}$ Syringe Luer-Lok Tip ${ }^{\circledR}$, Gloucester, UK) was used to inject the Botox type I (Allergan, Westport, Co. Mayo, Ireland) at 2.5 units per $0.1 \mathrm{~mL}$ diluted in $1 \mathrm{~mL} 0.9 \%$ sodium chloride solution according to the recommended guidelines for dilution and storage. ${ }^{9}$ The solution was injected $3 \mathrm{~mm}$ lateral to the alar-facial groove at the level of the nostril opening at the insertion of the levator labii superioris alaeque nasi muscle.

\section{Postinjection}

After the injection, the patient was instructed to avoid lying down, exercising, or massaging the injection site for 4 hours. ${ }^{3}$ Two weeks later, postinjection photos were taken at the same position of the preinjection.

\section{Measuring the Improvement of Gummy Smile}

The pre- and postinjection photos of each case were uploaded to the SketchUp program (Autodesk Inc., Rafael, CA, 2015) to calculate the improvement in gingival display. The distance between the lower margin of the upper lip to the incisal edge of the upper central incisor was measured for each case before and after receiving the injection. The amount of improvement was calculated by:

Amount of improvement $=$ (The distance between the lower margin of the upper lip to the incisal edge before the injection - The distance between the lower margin of the upper lip to the incisal edge after the injection)/The distance between the lower margin of the upper lip to the incisal edge before the injection $\times 100)($ Fig. 1). 

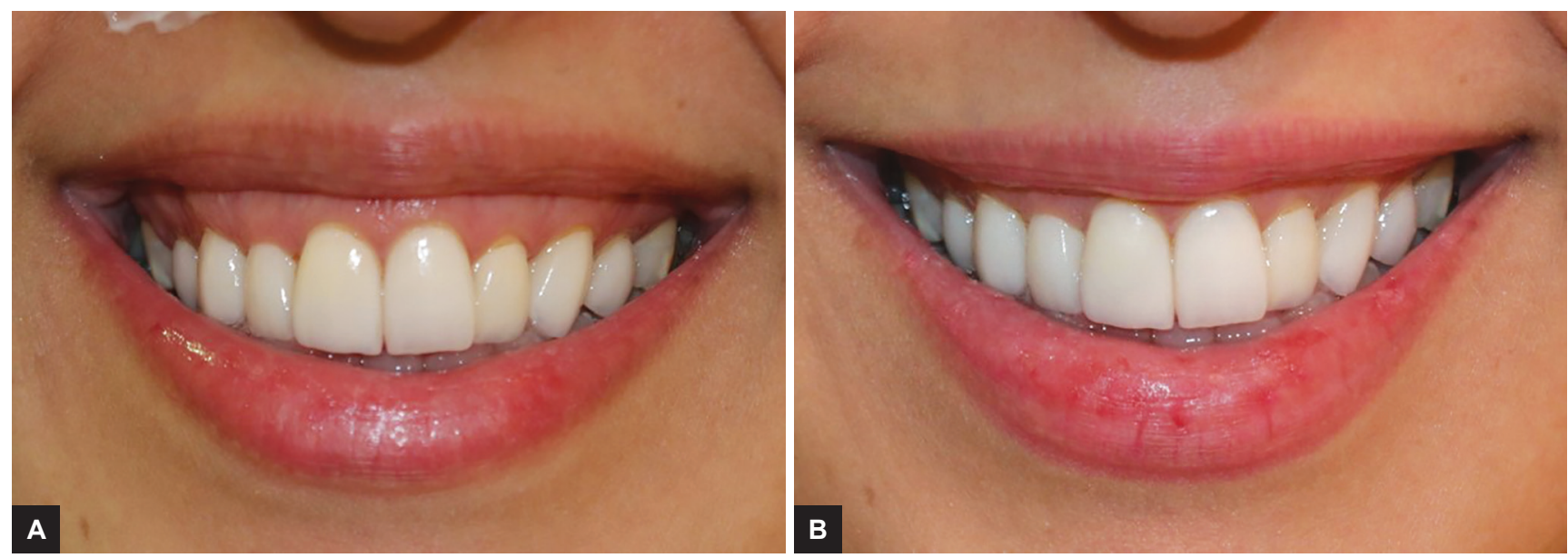

Figs $1 A$ and $B$ : Pre $(A)$ and post $(B)$ photos for a case with gummy smile treated by Botox

\section{Statistical Analysis}

Data were collected and analyzed using Statistical Package for the Social Sciences software (version 12, Chicago, Illinois, USA). Descriptive statistics were used to describe the mean percentage of the improvement in gummy smile.

\section{RESULTS}

The improvement in the gummy smile was shown 2 weeks after Botox injection. A significant decrease in the amount of gingival display was noticed before and after receiving the injection. The average improvement $(n=23)$ was 99.65\% (Table 1). No adverse effect was reported.

Table 1: Amount of improvement for each case with the total mean

\begin{tabular}{ll}
\hline Case number & Amount of improvement (\%) \\
\hline 1 & 100 \\
2 & 40.15 \\
3 & 142.11 \\
4 & 100 \\
5 & 139.76 \\
6 & 133.42 \\
7 & 128.24 \\
8 & 100 \\
9 & 123.43 \\
10 & 113.43 \\
11 & 100 \\
12 & 105.18 \\
13 & 100 \\
14 & 88.84 \\
15 & 100 \\
16 & 40.15 \\
17 & 40.15 \\
18 & 28.03 \\
19 & 100 \\
20 & 134.35 \\
21 & 131.35 \\
22 & 163.62 \\
23 & 41.93 \\
Mean & 99.65 \\
\hline & \\
\hline
\end{tabular}

\section{DISCUSSION}

Smile is the key to nonverbal communication..$^{10}$ The two important stages in the development of a smile are first when the upper lip raises to the nasolabial fold and inserts at the upper lip. Then, the medial muscle bundles will raise the lip at the anterior teeth, and the lateral muscle groups raise the lips at the posterior teeth. Cheek fat will resist the lips at the nasolabial fold. During the second stage, the lip will raise superiorly by three muscle groups: (i) Levator labii superioris, (ii) zygomaticus major, and the (iii) superior fibers of buccinator. ${ }^{11}$ The smile consists mainly of the framework of the lips, teeth, and the scalloped gingival border. The smile could also be divided into low, medium, and high depending on the lip position. According to Ezquerra et $\mathrm{al}^{12}{ }^{12}$ the smile is considered to be low when only part of the teeth are seen below the upper lip. It is medium when 1 to $3 \mathrm{~mm}$ of the gum is visible, and it is high when more than $3 \mathrm{~mm}$ of the gingiva is shown. It has been demonstrated that people with 0 to $2 \mathrm{~mm}$ of gingival exposure have the most attractive smile. ${ }^{13}$ In another study by Peck et al, ${ }^{11}$ the approximate percentage of smile types in the population illustrated that people with a low smile line are $<75 \%$, while the medium or average smile was approximately 75 to $100 \%$. In the same study, the authors calculated that gender plays a role in smile categories. The low smile line was found to occur more in males compared with females at a ratio of 2.5:1. According to Peck et al, ${ }_{11}^{11}$ this is the least preferable smile. On the contrary, a high smile line was found more in females at a ratio of 2:1. Silberberg et $\mathrm{a}^{10}$ found in their study that excessive gingival display occurs in approximately $10 \%$ of the population. This is in agreement with our study sample. The peak age for affected patients is between 20 and 30 years and it gradually decreases with age as the upper lip drops and minimizes gingival exposure. A gingival smile or gummy smile is due to (i) excessive vertical maxilla, (ii) hyperfunction of 
the lip elevator, and (iii) delayed passive eruption. The first is diagnosed through assessment of the smile skeletal components. The second is confirmed when skeletal analysis of maxilla shows normal dimensions, but $2 \mathrm{~mm}$ of the gingiva is visible when smiling. Diagnosis of the last group occurs when a large part of the dental crown is covered by the gingiva due to abnormal teeth development. ${ }^{12}$ An accurate examination should be performed to provide a correct diagnosis. A gummy smile needs to be diagnosed via a facial examination in which facial symmetry and proportions are measured in both the frontal and lateral views. ${ }^{10}$ Treatment methods have been developed and modified based on the different causes of a gummy smile. For example, if there is a skeletal imbalance, then orthognathic surgeries and orthodontic intervention will be suitable solutions. In addition, delayed passive eruption could be treated by periodontic intervention. Finally, muscular hyperfunction was usually treated by plastic reconstructive surgery and has only recently been treated by Botox injections. ${ }^{10}$

With the spread of esthetic dentistry, many patients are looking forward to obtaining an ideal smile via the least harmful methods. The ideal attractive smile components were studied by Silberberg et $\mathrm{al}^{10}$ and summarized as (i) minimal gingival display, (ii) symmetric display and harmony between the maxillary gingival line and upper lip, (iii) healthy gingival tissue filling the entire interproximal spaces, (iv) harmony between the anterior and posterior segments, (v) teeth in the correct anatomy and proportion, (vi) proper color and shade of the teeth, (vii) and a lower lip parallel to the incisal edges of the maxillary anterior teeth and to the imaginary line going through the contact points. A gummy smile is one of the most embarrassing situations a patient can have. Previous surgical treatments, such as LeFort I osteotomy, bone resection, crown lengthening, and gingivectomy were not favored by the patients due to their high morbidity, cost, and time. For example, LeFort I could affect nasal airway function in severe cases. ${ }^{5}$ In addition, orthodontic treatment was provided to treat a gummy smile caused by intruding incisors. ${ }^{3,5}$ The introduction of Botox type I to cosmetic dentistry was of great value. ${ }^{14}$ Multiple types of Botox exist that are produced by different strains of Clostridium botulinum (Botox A, B, C, D, E, F, and G). The only approved toxins for human use are types I, II, V, VI, and VII. However, Botox A and B are the only ones used as drugs, and type I is mainly used for esthetics. Many therapeutic treatments have been approved by the FDA with Botox, such as treating eye muscle problems, neck problems, and excessive sweating. ${ }^{15-17}$

Botox has been recently considered one of the first treatment options for gummy smile because of its easy and safe application, low risk, and reversible effects.
Perhaps most importantly, it has a rapid cosmetic effect. ${ }^{3}$ On the contrary, Botox lasts between 4 and 6 months depending on muscle activity and thickness. The authors reported that there was a significant difference in the amount of improvement of gummy smile, which is in agreement with Durgekar et al. ${ }^{16}$

As Peck et $\mathrm{al}^{11}$ mentioned in their study, gummy smile patients have $20 \%$ greater perioral muscular activity compared with the normal population, which will cause faster relapse. ${ }^{3}$ In this study, there is no adverse effect reported. In some cases, an improper injection technique will cause an unnatural asymmetric stretched lip appearance. ${ }^{9}$ On the contrary, Botox might affect the pronunciation of a few letters; cause some asymmetry in the upper lip, and difficulty kissing as reported by Sandler et al. ${ }^{17}$

\section{CONCLUSION}

Botox type I is an effective temporary conservative technique to improve gummy smile. It improves both gummy smile and the involved muscle. Botox type I is safe to correct the gingival display when used at an appropriate dose and setting.

\section{CLINICAL SIGNIFICANCE}

This study has shown that Botox type I was effective in treating patients who were suffering from gummy smile. The gummy smile was affecting patients' self-esteem, daily practice, and quality of life. With the immediate temporary solution, patients were satisfied about the provided treatment.

\section{REFERENCES}

1. Polo M. Botulinum toxin Type A in the treatment of excessive gingival display. Am J Orthod Dentofacial Orthop 2005 Feb;127(2):214-218.

2. Jaspers GW, Pijpe J, Jansma J. The use of botulinum toxin Type A in cosmetic facial procedures. Int J Oral Maxillofac Surg 2011 Feb;40(2):127-133.

3. Mazzuco R, Hexsel D. Gummy smile and botulinum toxin: a new approach based on the gingival exposure area. J Am Acad Dermatol 2010 Dec;63(6):1042-1051.

4. Patel DP, Thakkar SA, Suthar JR. Adjunctive treatment of gummy smile using botulinum toxin Type-A (case report). J Dent Med Sci 2012 Nov-Dec;3(1):22-29.

5. Indra AS, Biswas PP, Vineet VT, Yeshaswini T. Botox as an adjunct to orthognathic surgery for a case of severe vertical maxillary excess. J Maxillofac Oral Surg 2011 Sep;10(3):266-270.

6. Flynn TC. Advances in the use of botulinum neurotoxins in facial esthetics. J Cosmet Dermatol 2012 Mar;11(1):42-50.

7. Bansal N, Singla J, Gera G, Gupta M, Kaur G. Reliability of natural head position in orthodontic diagnosis: a cephalometric study. Contemp Clin Dent 2012 Jul;3(2):180-183.

8. Sam, E.; Marie, J.; Ruth, B. Clinical photography, art, graphics and video in health care. IMI National Guidelines, Orthodontic Photography. London: Institute of Medical Illustrators; 2008. 
9. Klein AW. Dilution and storage of botulinum toxin. Dermatol Surg 1998 Nov;24(11):1179-1180.

10. Silberberg N, Goldstein M, Smidt A. Excessive gingival display - etiology, diagnosis, and treatment modalities. Quintessence Int 2009 Nov-Dec;40(10):809-818.

11. Peck S, Peck L, Kataja M. The gingival smile line. Angle Orthod 1992 Summer;62(2):91-100.

12. Ezquerra F, Berrazueta MJ, Ruiz-Capillas A, Arregui JS. New approach to the gummy smile. Plast Reconstr Surg 1999 Sep;104(4):1143-1150.

13. Hunt $O$, Johnston $C$, Hepper P, Burden D, Stevenson M. The influence of maxillary gingival exposure on dental attractiveness ratings. Eur J Orthod 2002 Apr;24(2):199-204.
14. Sepehr A, Chauhan N, Alexander AJ, Adamson PA. Botulinum toxin type a for facial rejuvenation: treatment evolution and patient satisfaction. Esthetic Plast Surg 2010 Oct;34(5): 583-586.

15. de Maio, M.; Rzany, B. Botulinum toxin in esthetic medicine. Berlin, Heidelberg: Springer Science \& Business Media; 2007.

16. Durgekar SG, Nagaraj K, Naik V. The ideal smile and its orthodontic implications. World J Orthod 2010 Fall;11(3): 211-220.

17. Sandler PJ, Alsayer F, Davies SJ. Botox: a possible new treatment for gummy smile. Virtual J Orthod 2007 Feb;7(4): 30-34. 